\title{
TREINTA AÑOS DE MANEJO DE RENOVALES DE ROBLE UN ANÁLISIS DE SU RESPUESTA EN CRECIMIENTO Y ESTRUCTURA
}

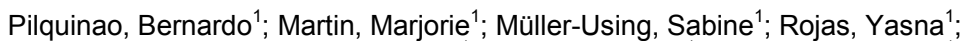 \\ Villalobos, Enrique ${ }^{1}$; Guiñez, Rodrigo ${ }^{1}$ y Barrientos, Marco ${ }^{1}$.
}

\section{RESUMEN}

Los renovales de roble (Nothofagus obliqua (Mirb.) Oerst.) del sur de Chile se distribuyen preferentemente en sitios de buena calidad y presentan en general buena accesibilidad. Muchos de estos renovales han sido intervenidos mediante programas de incentivos para el manejo sustentable, con el objetivo de incrementar el valor de estos. No obstante, se desconoce si finalmente este objetivo se cumplió, y si realmente existió alguna respuesta favorable a las intervenciones (raleos) que estos rodales han tenido en el transcurso de su rotación.

El presente estudio se orientó a evaluar estos aspectos, y para esto se planteó como objetivo caracterizar la composición, calidad y sanidad a los renovales de roble, que han sido sujetos a uno o más raleos comerciales, con énfasis en la participación de los árboles futuro y evaluar la relación entre la respuesta al raleo y la oportunidad de intervención de los rodales.

El estudio se desarrolló en veinte rodales ubicados entre las regiones de La Araucanía y Los Ríos, los cuales tuvieron la última intervención, al menos en los últimos 10 años, mediante un criterio comercial. Los rodales se distribuyeron aproximadamente entre los $39^{\circ} 07^{\prime} 53^{\prime \prime}$ y los $40^{\circ} 11^{\prime} 57^{\prime \prime}$ de Latitud Sur y los $72^{\circ} 51^{\prime} 46^{\prime \prime}$ y $72^{\circ} 00^{\prime} 37^{\prime \prime}$ de Longitud Oeste y a una altitud entre los 80 y $720 \mathrm{msnm}$.

En cada uno de los rodales se estableció un conglomerado compuesto por tres parcelas circulares de $500 \mathrm{~m}^{2}$. Se midieron las variables de estado de los árboles individuales (especie, DAP, altura total, altura comienzo de copa, diámetro de copas, estado sanitario, identificación de árboles futuro, entre otros). Se determinó el crecimiento de los rodales a través de la extracción de tarugos de diez individuos pertenecientes al estrato superior de cada rodal.

Los resultados determinaron que, en promedio, los rodales mantienen 160 árboles futuro. Según el diagrama de manejo de densidad para la especie, esta cantidad de árboles formarían la fase final de la rotación, cuando se considera una cosecha con 40-45 cm de diámetro. Las tasas máximas de crecimiento corriente (ICA) del presente estudio varío entre 0,82 a 1,06 cm/año. En tanto, el crecimiento promedio (IMA) para todos los rodales estuvo entre los 0,41 y $0,71 \mathrm{~cm} / a$ ño.

En general, los rodales muestreados respondieron a las intervenciones realizadas. Los crecimientos corrientes máximos se presentaron alrededor de los 12 años, en los distintos rodales medidos y los IMA máximos bordean los 20 años. Ello permite establecer este rango como el óptimo para realizar las primeras intervenciones en renovales de roble. Una intervención temprana, a pesar de no ser atractiva desde el punto de la extracción de volumen maderero e ingreso económico, sin duda determinará una mejor tasa de crecimiento futura del rodal.

Palabras clave: Renovales de roble, Raleos, Crecimiento, Manejo silvícola.

\footnotetext{
${ }^{1}$ Investigadores Instituto Forestal, Sede Los Ríos. Valdivia Chile. bpilquinao@infor.cl
} 


\section{SUMMARY}

Second growth forests of roble (Nothofagus obliqua (Mirb.) Oerst.) in southern Chile are mainly distributed in good quality sites and generally have good accessibility. Many of these second growth forests have been intervened through incentive programs for sustainable management, with the aim of increasing the value of sustainable management. However, the results of these interventions are still unknown, and whether there was any favorable response to the interventions (thinning) that these stands have had in the course of their rotation.

The aim of this study was to assess these aspects. The main objective was to characterize the composition, quality and health condition of stands, with one or more commercial thinnings. Evaluating the participation of future trees and assessing the relationship between the thinning response and the opportunity for intervention.

The study included twenty stands located between the Regions of La Araucanía and Los Ríos, which had the last intervention, at least in the last 10 years, under a commercial criterion. The stands were distributed approximately between 39-07'53" and 40-11'57" South and 72-51'46" and 72-00'37" West and at an altitude between 80 and 720 masl. On each of the stands, a cluster composed of three nested circular plots of $500 \mathrm{~m}^{2}$ was established. Individual tree state variables (species, DAP, total height, crown height, crown diameter, tree health status, future tree identification, among others) were measured. The growth of the stands was determined through the extraction of cores from ten individuals belonging to the upper stratum of each stand.

The results found that, on average, stands maintain 160 future or selected trees. According to the density management diagram for the species, this number of trees would form the final phase of rotation, when considered a harvest at $40-45 \mathrm{~cm}$ in diameter. The diameter maximum rates of current annual increment $(\mathrm{CAI})$ of this study were between 0.82 and $1.06 \mathrm{~cm} /$ year. Meanwhile, average growth or mean annual increment (MAI) for all stands was between 0.41 and $0.71 \mathrm{~cm} /$ year. In general, the sampled stands did respond to the interventions. It was found that maximum current growths were around the age of 12, at different measured stands and, maximum MAI is around 20 years. This makes it possible to further establish this range as optimal for performing the first interventions in roble's second growth forests. Early intervention, despite not being attractive from the point of timber volume extraction and economic income, will certainly determine a better future growth rate of the stand.

Keywords: Roble, Second growth forest, Thinning, Growth, Forest management. 


\section{INTRODUCCIÓN}

La especie roble (Nothofagus obliqua) se caracteriza por la alta calidad de su madera en términos estructurales y visuales (Juacida et al., 2000). A pesar de ello y de la gran superficie de 1,5 millones de hectáreas que cubren los bosques secundarios de esta especie (CONAF, 2017), en la actualidad solo ocupan un pequeño nicho en el mercado de la madera en Chile (INFOR, 2018). Los bosques de roble se distribuyen mayormente en sitios de buena calidad y presentan en general buena accesibilidad (Müller-Using et al., 2012). Forman parte del Tipo Forestal Roble-Raulí-Coigüe, el cual se considera dentro de los bosques que son foco de las metas de manejo con fines madereros, propuestas por la Política Forestal, donde se espera que al año 2035, un millón de hectáreas de bosques nativos sean incorporadas al manejo forestal sustentable con fines de producción de bienes de alto valor.

En apoyo de este desafío, el Estado chileno cuenta con programas de incentivo al manejo de bosque nativo, como un instrumento para incrementar el valor de estos (Ley $\mathrm{N}^{\circ} 20.283$ ). Estos programas son la continuidad de un proyecto de cooperación internacional iniciado en el año 1998 (Decreto 1722), el cual implementó en Chile un programa de manejo de renovales a través de raleos, generalmente por lo alto y concentrado en la promoción de árboles futuro (FAO 2016). La mayoría de estos bosques que hoy se encuentran entre los 40 y 80 años de edad (Grosse y Quiróz, 1999), han sido intervenidos a través de estos programas de manejo sustentable.

Los datos de ensayos silvícolas son sin duda fundamento importante de cualquier política forestal, dado que entregan el potencial de crecimiento de los bosques y las técnicas como alcanzarlos. No obstante, existe la posibilidad de que, en un contexto de manejo comercial, se generen desviaciones de estas situaciones controladas y que el impacto de los programas de incentivo en la calidad de los bosques también sea distinto a lo esperado. Por ejemplo, parte importante de los bosques de segundo crecimiento no han sido intervenidos tempranamente a través de raleos o podas y presentan limitaciones de calidad (Gezan et al., 2007) y, por su edad más avanzada, ya se encuentran fuera de su fase de crecimiento óptimo, que para roble se da en un rango de edad de 15 años (Grosse et al., 2007).

Para aportar antecedentes a esta hipótesis, el presente estudio evalúa como han reaccionado los rodales de propietarios privados, los cuales han sido intervenidos al menos en los últimos 10 años, en un contexto de manejo comercial de bosque en la región de Los Ríos y el sur de la región de la Araucanía.

\section{OBJETIVO}

El objetivo principal escaracterizar la composición, calidad y sanidad a los renovales de roble, que han sido sujetos a uno o más raleos comerciales, con énfasis en la participación de los árboles futuro y evaluar la relación entre la respuesta al raleo y la oportunidad de intervención de los rodales.

\section{MÉTODODOLOGÍA}

\section{Área de estudio}

El área de estudio se encuentra localizada en la Depresión Intermedia y Precordillera de los Andes de la región de Los Ríos y el sur de la región de La Araucanía (Provincia de Cautín), aproximadamente entre los $39^{\circ} 07^{\prime} 53^{\prime \prime}$ y los $40^{\circ} 11^{\prime} 57^{\prime \prime}$ de Latitud Sur y los $72^{\circ} 51^{\prime} 46^{\prime \prime}$ y $72^{\circ} 00^{\prime} 37^{\prime \prime}$ de 
Longitud Oeste y a una altitud entre los 80 y $720 \mathrm{msnm}$. El área presenta un clima templado lluvioso (Veblen et al., 1981), caracterizado por temperaturas moderadas y altas precipitaciones anuales que oscilan entre los 1.500 y $3.000 \mathrm{~mm}$. Los suelos se caracterizan por su origen de cenizas volcánicas recientes, comúnmente llamados trumaos, caracterizados por ser profundos y de buen drenaje (Peralta, 1976). Todas estas características determinan una zona de buen crecimiento para roble (Donoso et al., 1993).

\section{Selección y Medición de Unidades de Muestreo}

En base a la información proporcionada por propietarios y administradores de bosque nativo, se seleccionaron 20 rodales dominados por roble (Figura $\mathrm{N}^{\circ} 1$ ). Estos rodales debían presentar una estructura de renoval y haber sido intervenidos mediante raleos entre 5 y 10 años atrás.

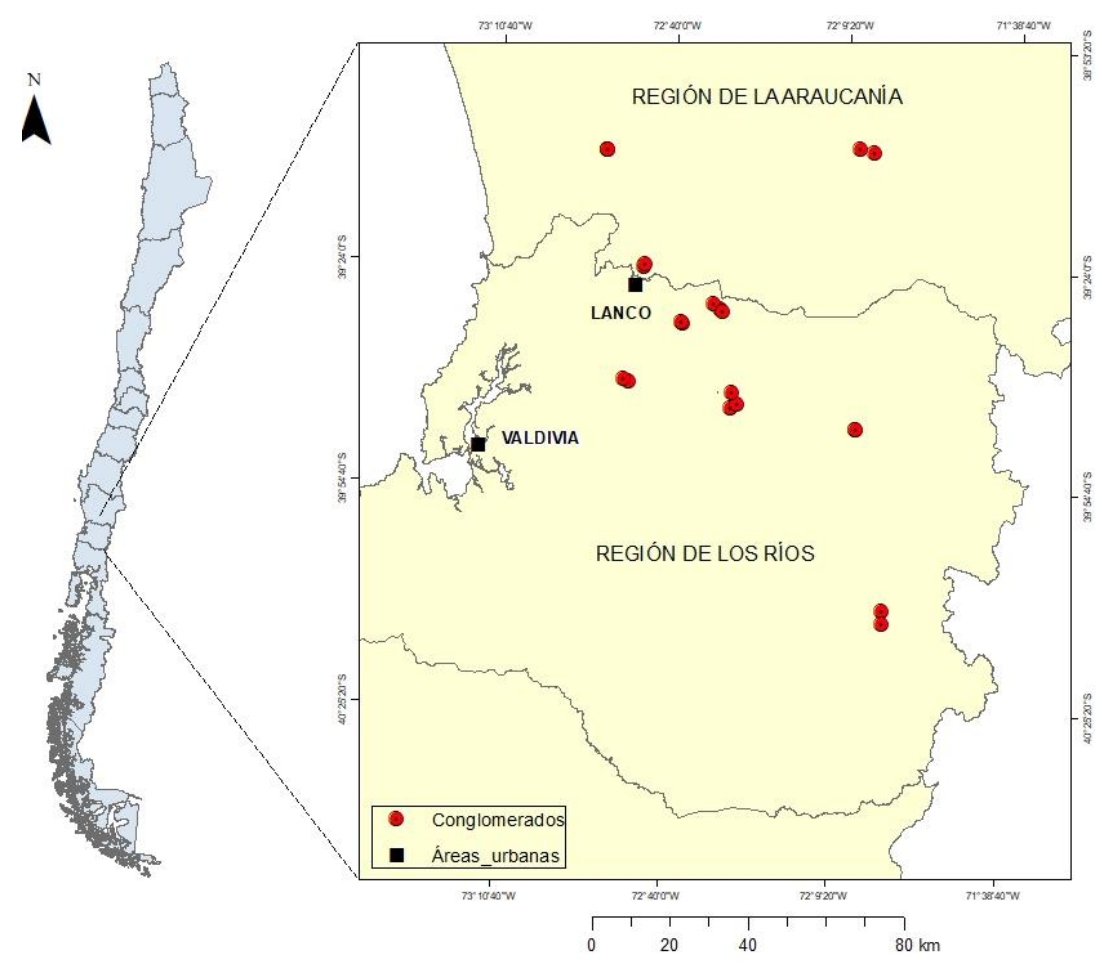

Figura $\mathrm{N}^{\circ} 1$

MAPA DE UBICACIÓN DE LOS RODALES

En cada uno de los rodales se estableció un conglomerado compuesto por tres parcelas circulares de $500 \mathrm{~m}^{2}$ cada una y distribuidas de acuerdo al procedimiento descrito por Martin et al., 
(2009). Las variables más importantes registradas fueron especie, DAP, altura total, altura comienzo de copa, diámetro de copas y estado sanitario. Además, en cada parcela se identificaron los árboles futuro, de acuerdo a las características de sanidad y forma, espaciamiento homogéneo, área de copa y cantidad de trozas madereras a obtener.

El estado sanitario de los individuos muestreados en los rodales, se evaluó a través de tres categorías:

- Sano: Sin daño o enfermedad aparente.

- Enfermo: El árbol tiene síntomas asociados a problemas prolongados en el tiempo, como ataque de insectos, bacterias $u$ hongos.

- Dañado: Tiene una connotación temporal más corta que la enfermedad. Ejemplo: ápices quemados a causa de las heladas, árboles quebrados por el efecto del viento, tallos comidos por roedores, otros.

El crecimiento de los árboles está relacionado con el tamaño de las copas. Una forma de evaluar esto es a través de la clase de copa según su posición sociológica en el rodal. Es por ello que se analizó la frecuencia de las clases de copa según la metodología del Inventario Forestal Nacional (Martin et al., 2009) en cada uno de los rodales. Así, se tienen las siguientes clases de copa.

Dominantes: Árboles cuyas copas se desarrollan por sobre el nivel general de las copas de los demás árboles del rodal y reciben luz del sol completa desde arriba y parcialmente desde los lados.

- Codominantes: Árboles cuyas copas se encuentran al nivel general de las copas del dosel. Las copas reciben luz completa desde arriba, pero escaza luz penetra desde los costados.

- Intermedios: Estos son árboles más bajos en altura que los dominantes y codominantes. Reciben poca luz directa desde arriba y nada desde sus costados.

- Suprimidos: Las copas de estos árboles se encuentran completamente bajo el nivel general del dosel y no reciben luz directa desde ninguna dirección.

La información dasométrica fue incorporada a una base de datos (SQL SERVER 2014), a partir de la cual se obtuvieron los principales parámetros de rodal como número de árboles por hectárea (árboles/ha), área basal $\left(\mathrm{m}^{2} / \mathrm{ha}\right)$, volumen $\left(\mathrm{m}^{3} \mathrm{ssc} / \mathrm{ha}\right)$, diámetro medio cuadrático (DMC en $\mathrm{cm})$ y altura media $(\mathrm{m})$.

Los individuos muestreados se clasificaron también respecto a la forma que presenta el fuste, la cual depende de factores como la especie, el sitio, el manejo realizado y la densidad del rodal. De este modo se tienen las siguientes categorías: recto, bifurcado, inclinado, curvado, torcido y multifustal.

Respecto de los árboles futuro, estos tienen que reunir las siguientes características: recto, sano, buena calidad, con potencial maderero y pertenecer al dosel superior. 


\section{Evaluación del Crecimiento}

Para el análisis del crecimiento, se tomó una muestra a 10 árboles de roble por rodal. Los árboles seleccionados debían presentar características de árbol futuro (en el caso de no contar con suficientes muestras con estas características, se seleccionaron algunos individuos del dosel intermedio). De cada árbol se extrajeron dos tarugos a la altura del DAP, en direcciones norte-sur y este-oeste y fueron montados usando técnicas estándar para su pulido y medición con el fin de determinar el incremento anual y la edad.

Se asignó el año a cada ancho del anillo y se determinó la edad de cada individuo muestreado, considerando el año 2018 como último anillo completo. En total se procesaron tarugos provenientes de 194 árboles, descartándose aquellos individuos a los que, por problemas de daño del tarugo, no se pudieron medir.

Dado que no se conoce todo el historial de manejo de los rodales, se recurrió a métodos utilizados en estudios de la dinámica forestal y disturbios (Abrams et al., 2001; Black and Abrams, 2003; Lorimer and Frelich, 1989; Trotsiuk et al., 2018), a fin de reconstruir intervenciones pasadas.

Los posibles disturbios a nivel de dosel se analizaron mediante el método propuesto por Nowacki and Abrams (1997) y utilizando el criterio del crecimiento radial promedio. Esta técnica utiliza como medio de evaluación el cambio relativo del crecimiento en radio observado antes y después de un determinado año. Para el análisis de las respuestas temporales se tomó como referencia un promedio móvil del crecimiento de 5 años antes de las intervenciones y 5 años posterior a las intervenciones. Para ello se utilizó la siguiente expresión:

$$
\mathrm{GC}=[(\mathrm{M} 2-\mathrm{M} 1) / \mathrm{M} 1]
$$

Donde:

GC: Tasa de cambio en el crecimiento

M1: Crecimiento medio de los cinco años precedentes

M2: Crecimiento medio de los cinco subsiguientes

Un umbral mínimo de liberación se considera moderado con un 25\% (tasa de 0,25) de cambio en el crecimiento y una liberación mayor para un valor sobre $>50 \%$ (tasa de 0,5 ) del cambio en crecimiento. Para considerar que un cierto rodal fue sometido a algún tipo de raleo, se asumió que al menos 3 de los diez árboles (o 2 en caso de los seis) aumentaran sus tasas en los mismos años.

Este método tiene como ventaja que es de amplia aplicación, aún en muestras pequeñas y no requiere información de la autoecología de la especie. Tiene la desventaja de que puede identificar falsas liberaciones o no identificar las correctas (Black and Abrams, 2003; Fraver and White, 2005). No obstante, y dado que en este trabajo se representan mayoritariamente árboles dominantes, se considera que la muestra evita estas falsas liberaciones.

Para el análisis se utilizó el programa estadístico R, específicamente el paquete TRADER (Altman et al., 2014). Además, a partir de los anchos de anillos se calcularon los incrementos medios e incrementos corrientes anuales en diámetro de los árboles dominantes de los rodales. El incremento anual corriente (ICA) corresponde al crecimiento de un individuo en el periodo de un año y el incremento medio anual (IMA) corresponde al valor del DAP alcanzado hasta una edad determinada dividido por esa edad. 
La edad de culminación biológica del crecimiento se produce en el punto en que se cruzan las curvas del ICA y del IMA (Drake et al., 2003) y para la producción de trozas es el momento de máximo crecimiento. Cuando se opta por productos de mayor valor, los raleos se debiesen efectuar en la fase de crecimiento juvenil (Assmann, 1970), que es más cercano al descenso de la curva del ICA, así lo mas importante es el crecimiento del árbol individual y no necesariamente la producción de la masa (Lavery, 1986).

\section{RESULTADOS}

\section{Características de los Rodales}

En el Cuadro $\mathrm{N}^{\circ} 1$ se muestra la caracterización de los rodales muestreados. El rango de edad media de los rodales abarca entre 34 y 71 años, encontrándose un $70 \%$ de rodales sobre los 60 años. De acuerdo a los valores de DMC, el $40 \%$ de los rodales muestreados se encuentran en un estado de desarrollo de fustal joven, el $45 \%$ corresponden a latizal alto y el $15 \%$ a latizal bajo (CONAF, 2018). El rango volumétrico de los rodales es altamente variable, abarcando entre los 150 y $700 \mathrm{~m}^{3} \mathrm{ssc} / \mathrm{ha}$. Según estos datos el IMA calculado se encuentra entre 4,8 y $9,9 \mathrm{~m}^{3} /$ ha/año, solo dos rodales salen de este rango con valores menores de 2,3 y $2,8 \mathrm{~m}^{3} / \mathrm{ha} / \mathrm{año}$.

\section{Cuadro $\mathrm{N}^{\circ} 1$
PARÁMETROS DE RODAL DE LOS RODALES MUESTREADOS}

\begin{tabular}{|c|c|c|c|c|c|c|c|}
\hline Rodal & Comuna & $\begin{array}{c}\text { AB } \\
\left(m^{2} / h a\right)\end{array}$ & $\begin{array}{c}\text { Densidad } \\
\text { (arb/ha) }\end{array}$ & $\begin{array}{l}\text { Volumen } \\
\text { (m³ss/ha) }\end{array}$ & $\begin{array}{l}\text { DMC } \\
(\mathrm{cm})\end{array}$ & $\begin{array}{c}\text { Edad } \\
\text { (años) }\end{array}$ & $\begin{array}{c}\text { IMA } \\
\text { volumen } \\
\left(\mathrm{m}^{3} / \mathrm{ha} / \mathrm{año} \text { ) }\right.\end{array}$ \\
\hline Alaska 1 & Máfil & 30,9 & 427 & 404,6 & 30,4 & 60 & 6,7 \\
\hline Alaska 2 & Máfil & 19,1 & 533 & 185,3 & 21,4 & 66 & 2,8 \\
\hline Arquilhue 1 & Futrono & 39,2 & 993 & 372,3 & 22,5 & 63 & 5,9 \\
\hline Arquilhue 2 & Futrono & 37,4 & 760 & 461,8 & 25,1 & 68 & 6,8 \\
\hline Curirruca 1 & Lanco & 37,2 & 760 & 378,5 & 25,1 & 70 & 5,4 \\
\hline Curirruca 2 & Lanco & 46,7 & 887 & 543,7 & 27,5 & 71 & 7,7 \\
\hline Curirruca 3 & Lanco & 13,5 & 573 & 159,5 & 17,2 & 69 & 2,3 \\
\hline Don Cristóbal & Villarrica & 45,0 & 480 & 644,7 & 34,8 & 68 & 9,5 \\
\hline El Dorado & Villarrica & 41,7 & 527 & 454,1 & 32,2 & 70 & 6,5 \\
\hline Miraflores1 & Lanco & 20,7 & 873 & 193,2 & 17,4 & 34 & 5,7 \\
\hline Miraflores2 & Lanco & 25,6 & 880 & 308,6 & 19,3 & 50 & 6,2 \\
\hline Nueva Etruria 1 & Pitrufquen & 29,2 & 340 & 322,7 & 33,3 & 67 & 4,8 \\
\hline Nueva Etruria 2 & Pitrufquen & 25,6 & 247 & 358,8 & 36,6 & 65 & 5,5 \\
\hline Porvenir 1 & Loncoche & 26,9 & 553 & 243,8 & 25,8 & 51 & 4,8 \\
\hline Porvenir 2 & Loncoche & 33,1 & 673 & 359,0 & 25,4 & 42 & 8,5 \\
\hline Puñir 1 & Panguipulli & 27,5 & 713 & 317,8 & 23,0 & 42 & 7,6 \\
\hline Puñir 2 & Panguipulli & 24,7 & 353 & 301,8 & 30,4 & 46 & 6,6 \\
\hline Santa Olga & Panguipulli & 42,8 & 647 & 491,0 & 29,2 & 63 & 7,8 \\
\hline Trafun 1 & Panguipulli & 47,0 & 667 & 702,6 & 31,1 & 71 & 9,9 \\
\hline Trafun 2 & Panguipulli & 33,8 & 367 & 465,0 & 35,1 & 65 & 7,2 \\
\hline
\end{tabular}


Al incorporar los parámetros de rodal al Diagrama de Manejo de Densidad desarrollado por INFOR (Müller-Using et al., 2012), se observa que más del $90 \%$ de los rodales se encuentran ubicados sobre la línea de cierre de copas (Línea B), y bajo los $55 \%$ de densidad relativa (Figura $\mathrm{N}^{\circ} 2$ ).

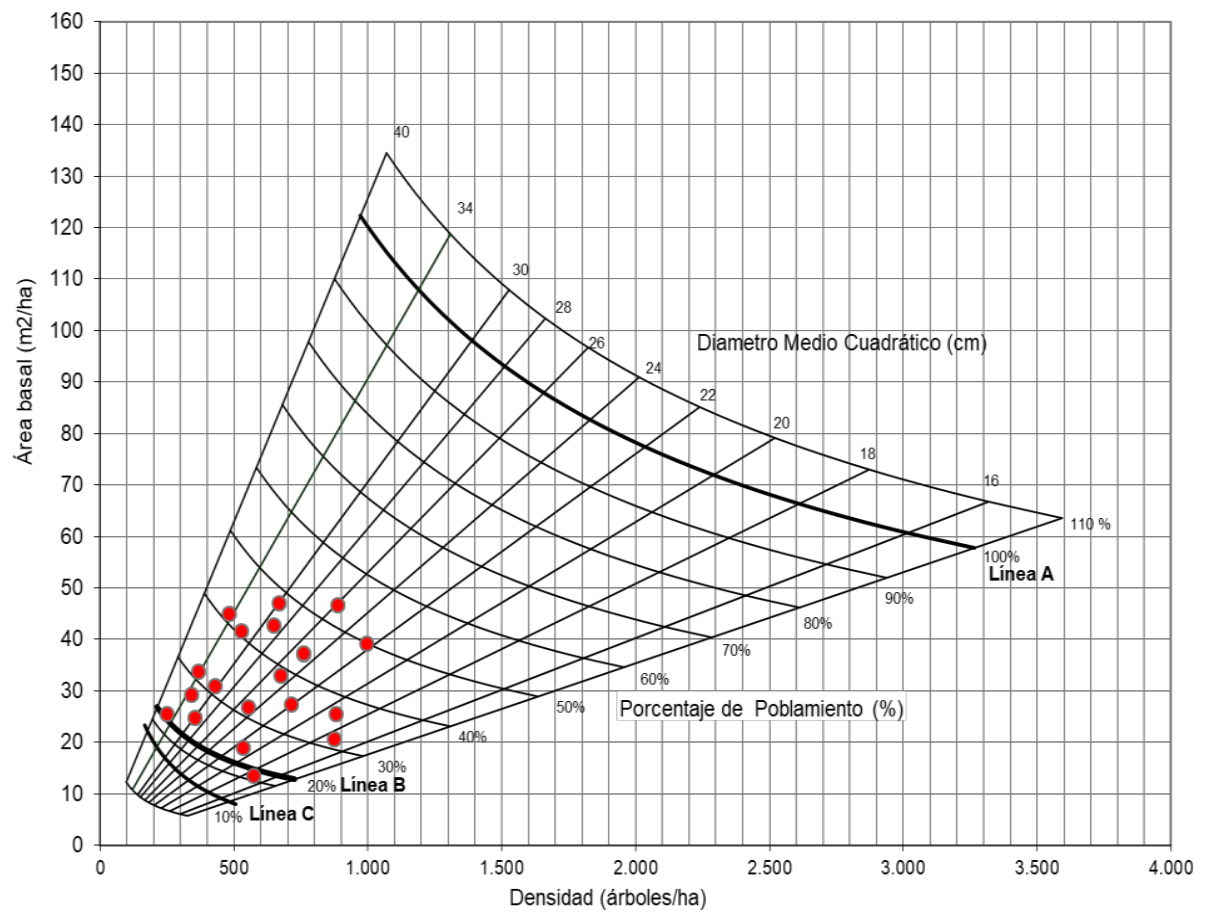

Figura $\mathrm{N}^{\circ} 2$

DENSIDAD RELATIVA DE LOS RODALES DE ROBLE

En el diagrama de densidad se visualizan los distintos estados de desarrollo de los rodales muestreados. Si esto expresa también el estado de desarrollo de los robles, o es resultado de una estructura irregular por la mezcla con especies siempreverdes, se puede observar en el Cuadro $\mathrm{N}^{\circ} 2$. Aquí se clasificaron los rodales en dos grupos de acuerdo a su DMC y respectivo estado de desarrollo según CONAF (2018). Así, 12 rodales se encuentran en estado de latizal bajo y alto, y 8 rodales en estado de fustal joven.

Respecto a la participación de la especie roble, se registraron cuatro rodales que estaban clasificados como latizal al incluir las especies acompañantes. No obstante, al considerar solo los robles, estos rodales cambian a un estado de desarrollo de fustal. Considerando exclusivamente a los robles y su incremento medio en DAP, se registran diferencias importantes en el incremento medio entre latizales y fustales. 
Los parámetros que permiten aumentar el valor maderero de un rodal y que se pretende mejorar a través de raleos, son principalmente la sanidad, la forma, el crecimiento de los árboles futuro y la regulación de la mezcla (Grosse y Villalobos 2009), la cual en este caso es lograr o mantener una participación alta de roble.

Lo anterior hace pensar que, para efectos de la planificación silvícola, los parámetros de rodal y principalmente el DMC debiera estar enfocado solo en los robles como especie principal, sin considerar las especies acompañantes, debido a que por ser de menor diámetro tienden a reducir los valore de DMC.

\section{Cuadro $\mathrm{N}^{\circ} 2$}

VALORES DE DMC DE ROBLE VERSUS DMC DE OTRAS ESPECIES

\begin{tabular}{|c|c|c|c|c|c|}
\hline $\begin{array}{l}\text { Estado de } \\
\text { desarrollo }\end{array}$ & Rodal & $\begin{array}{c}\text { DMC } \\
\text { rodal }(\mathrm{cm})\end{array}$ & $\begin{array}{c}\text { DMC robles } \\
\text { (cm) }\end{array}$ & $\begin{array}{l}\text { DAP Medio } \\
\text { robles }(\mathrm{cm})\end{array}$ & $\begin{array}{l}\text { IMA DAP } \\
\text { (cm/año) }\end{array}$ \\
\hline \multirow{12}{*}{ Latizal } & Miraflores 1 & 17,4 & 17,8 & 16,6 & 0,5 \\
\hline & Curirruca 1 & 25,1 & 32,9 & 32,7 & 0,5 \\
\hline & Curirruca 2 & 27,5 & 37,1 & 25,9 & 0,4 \\
\hline & Arquilhue 1 & 22,5 & 28,2 & 29,0 & 0,6 \\
\hline & Arquilhue 2 & 25,1 & 28,8 & 23,9 & 0,6 \\
\hline & Porvenir 1 & 25,8 & 29,3 & 19,6 & 0,3 \\
\hline & Puñir 1 & 23,0 & 24,2 & 19,1 & 0,4 \\
\hline & Alaska 2 & 21,4 & 22,2 & 15,2 & 0,2 \\
\hline & \begin{tabular}{|l|} 
Miraflores 2 \\
\end{tabular} & 19,3 & 21,0 & 35,8 & 0,6 \\
\hline & Curirruca 3 & 17,2 & 18,2 & 36,9 & 0,5 \\
\hline & Porvenir 2 & 25,4 & 31,3 & 30,8 & 0,4 \\
\hline & Santa Olga & 29,2 & 31,5 & 29,8 & 0,5 \\
\hline \multirow{8}{*}{ Fustal } & Alaska 1 & 30,4 & 35,8 & 37,3 & 0,5 \\
\hline & Trafún 1 & 31,1 & 37,7 & 34,3 & 0,5 \\
\hline & Trafun 2 & 35,1 & 35,0 & 35,7 & 0,5 \\
\hline & El Dorado & 32,2 & 35,5 & 36,6 & 0,5 \\
\hline & Don Cristobal & 34,8 & 37,7 & 30,9 & 0,7 \\
\hline & Puñir 2 & 30,4 & 30,7 & 30,2 & 0,7 \\
\hline & Nueva Etruria 1 & 33,3 & 39,7 & 38,4 & 0,6 \\
\hline & Nueva Etruria 2 & 36,6 & 38,1 & 37,3 & 0,6 \\
\hline
\end{tabular}

Respecto de su sanidad, los rodales mantienen un porcentaje superior al $80 \%$ de los individuos sin problemas sanitarios. Del total de rodales muestreados, 8 no tienen problemas sanitarios y los 12 rodales restantes tienen un porcentaje menor al $20 \%$ de individuos enfermos (Figura $\mathrm{N}^{\circ} 3$ ). 


\subsection{0}

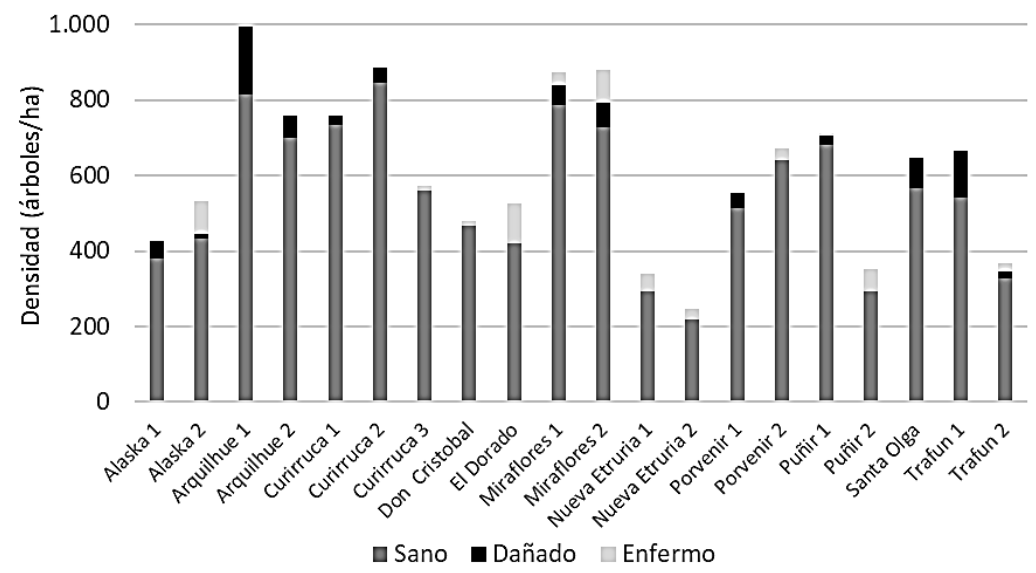

Figura $\mathrm{N}^{\circ} 3$

ESTADO SANITARIO DE LOS RODALES MUESTREADOS

Un $10 \%$ de los rodales muestreados (2 rodales) registraron más de un $50 \%$ de participación de individuos dominantes. En tanto, el $50 \%$ de los rodales (10 rodales) registraron solo un $30 \%$ de participación de los individuos dominantes en el rodal. (Figura $\mathrm{N}^{\circ} 4$ ). En general, en la mayoría de los rodales se observa una estructura estratificada que da cuenta de la evolución de la sucesión natural de los rodales y los efectos de los raleos.

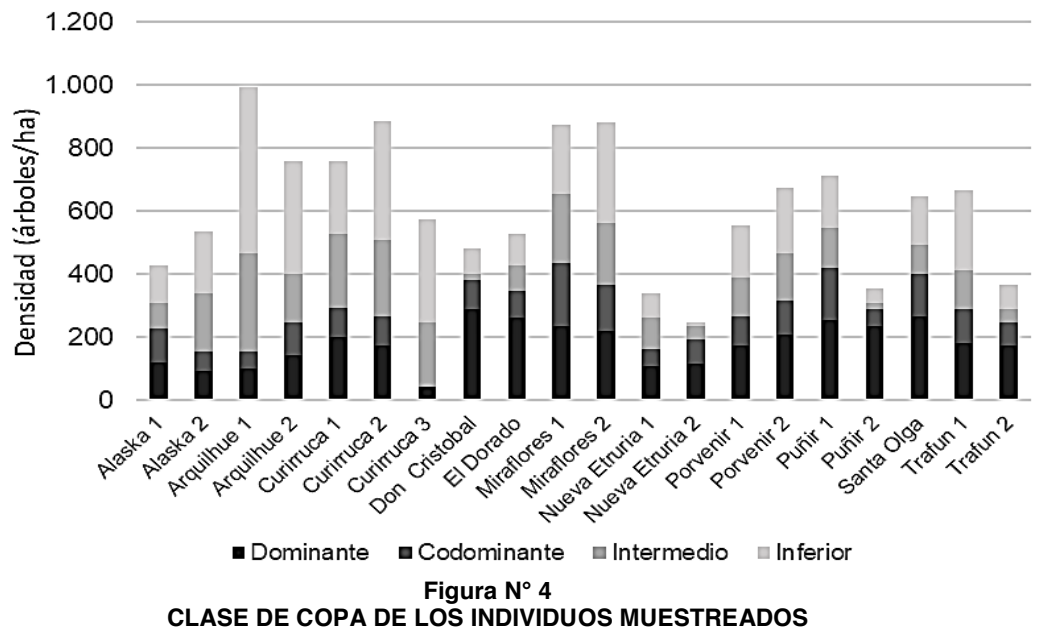


En la mayoría de los rodales, roble es la especie principal, pero todos los rodales tienen participación de otras especies. Como ya se mostró en el Cuadro $\mathrm{N}^{\circ} 2$, las especies secundarias en algunos casos se encuentran en un estrato inferior. Esto es reforzado por el hecho de que en promedio el $90 \%$ de las clases de copa dominante y codominante corresponden a individuos de roble (Figura $\mathrm{N}^{\circ} 5$ ).

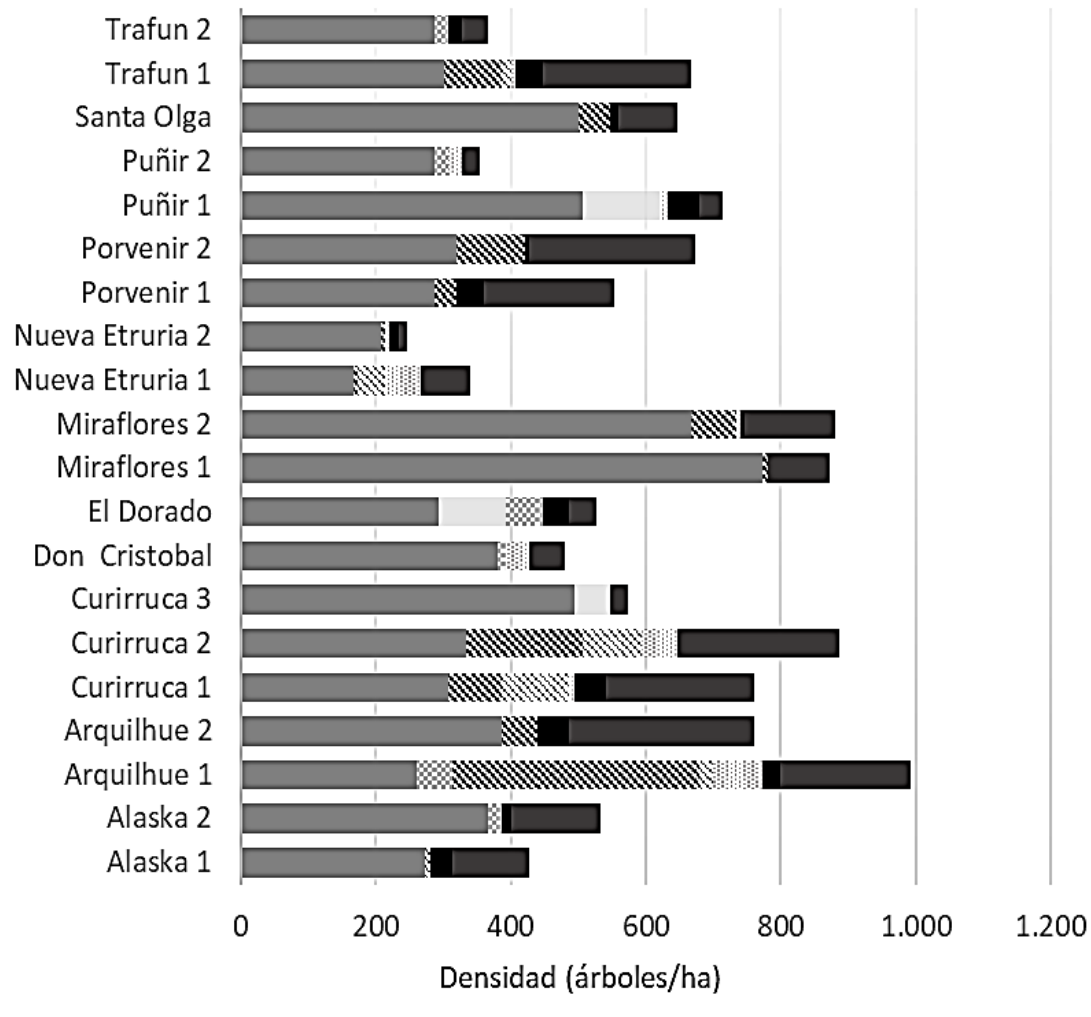

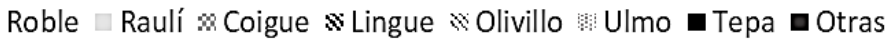

Figura $\mathrm{N}^{\circ} 5$

COMPOSICIÓN DE ESPECIES DE LOS RODALES DE ROBLE MUESTREADOS

La calidad del rodal y su futuro valor se puede representar en la cantidad de árboles que cumplieron los requisitos de un árbol futuro. La cantidad de árboles futuro fue bastante variable para cada rodal (Figura $N^{\circ} 6$ ). Así, tres rodales presentaron más del $60 \%$ de los individuos con características de árboles futuro, lo cual refleja el efecto positivo de los raleos y el buen manejo silvícola. En tanto, el $60 \%$ de los rodales (12 rodales) registraron una participación inferior al $30 \%$ de los individuos con características de árbol futuro. 
Cabe destacar, que dos rodales registraron un importante número de árboles futuro, con más de 300 árboles/ha, lo cual fue muy superior al promedio registrado por los otros rodales correspondiente a 142 árboles/ha.

No obstante, esto último, da cuenta de un porcentaje importante del $70 \%$ de los rodales que mantienen menos de 200 árboles futuro a nivel de hectárea. En este sentido, el manejo silvícola de los rodales muestreados está orientado a la liberación de estos árboles futuro, con el fin de concentrar en ellos el crecimiento y el volumen de madera aserrable al final de la rotación.

1.200

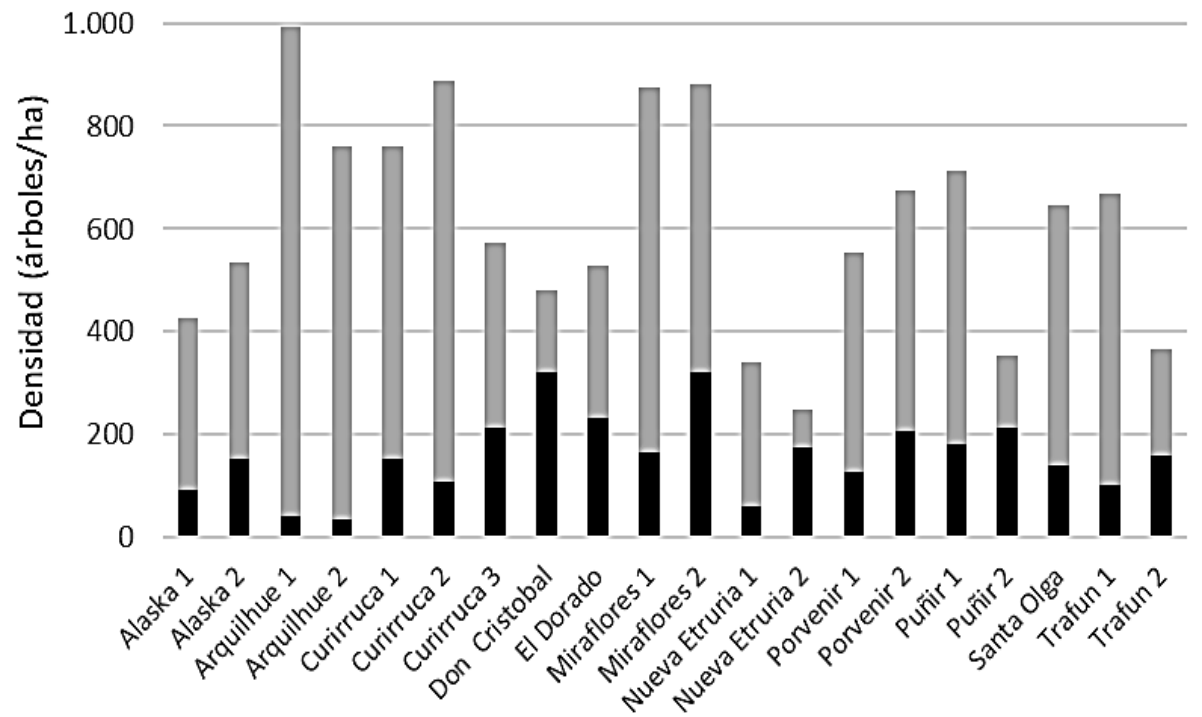

- Futuros $\square$ No futuros

\section{Figura $\mathrm{N}^{\circ} 6$ \\ ÁRBOLES FUTURO O CON POTENCIAL MADERERO}

Para definir la cantidad de árboles futuro necesarios para llegar a la cosecha con una ocupación de sitio completa, con árboles de buena calidad y maximizar así la producción de madera de valor, se puede usar el Diagrama de Manejo de Densidad de roble (Müller-Using et al., 2012).

Dado que el diámetro que se espera alcanzar en los rodales estudiados es de 40-45 cm, el número de árboles requeridos según esta herramienta es de alrededor de 200 individuos por hectárea. Este número disminuiría al aumentar el diámetro meta, asunto que amerita un análisis económico que trasciende esta publicación. 
En el Cuadro $\mathrm{N}^{\circ} 3$ se muestra en qué medida aportan los árboles futuro a los principales parámetros del rodal separados en estructura de latizales y fustales.

\section{Cuadro $\mathrm{N}^{\circ} 3$}

PARÁMETROS DASOMÉTRICOS DE RODAL DE LOS ARBOLES FUTURO

\begin{tabular}{|c|c|c|c|c|c|c|c|c|c|c|}
\hline $\begin{array}{c}\text { Estado } \\
\text { Desarrollo }\end{array}$ & Rodal & $\begin{array}{l}\text { DAP medio } \\
(\mathrm{cm})\end{array}$ & $\begin{array}{c}\text { Área basal } \\
\left(\mathrm{m}^{2} / \mathrm{ha}\right)\end{array}$ & $\begin{array}{c}\text { Área basal } \\
(\%)\end{array}$ & $\begin{array}{c}\text { Densidad } \\
\text { (arb/ha }\end{array}$ & $\begin{array}{l}\text { DMC } \\
\text { (cm) }\end{array}$ & $\begin{array}{c}\text { Volumen } \\
\left(\mathrm{m}^{3} / \mathrm{ha}\right)\end{array}$ & $\begin{array}{c}\text { Volumen } \\
\text { del total } \\
(\%)\end{array}$ & $\begin{array}{l}\text { IMADAP } \\
\text { (cm/año) }\end{array}$ & $\begin{array}{c}\text { IMA volumen } \\
\left(\mathrm{m}^{3} / \mathrm{ha} / / \mathrm{an} o\right)\end{array}$ \\
\hline \multirow{11}{*}{ Latizal } & Miraflores 1 & 23,8 & 7,8 & $38 \%$ & 167 & 24,1 & 80,2 & 42 & 0,7 & 2,4 \\
\hline & Curirruca 1 & 38,6 & 18,2 & $49 \%$ & 153 & 39,1 & 222,4 & 59 & 0,6 & 3,2 \\
\hline & Curirruca 2 & 46,7 & 18,8 & $40 \%$ & 107 & 47,5 & 266,1 & 49 & 0,7 & 3,7 \\
\hline & Arquilhue 1 & 45,9 & 7,3 & $19 \%$ & 40 & 46,2 & 100,7 & 27 & 0,7 & 1,6 \\
\hline & Arquilhue 2 & 39,5 & 12,8 & $34 \%$ & 100 & 40,4 & 192,7 & 42 & 0,6 & 2,8 \\
\hline & Porvenir 1 & 34,8 & 11,7 & $43 \%$ & 127 & 35,1 & 130,9 & 54 & 0,7 & 2,6 \\
\hline & Puñir 1 & 29,2 & 12,0 & $44 \%$ & 180 & 29,5 & 143,7 & 45 & 0,7 & 3,4 \\
\hline & Curirruca 3 & 19,3 & 9,0 & $67 \%$ & 213 & 23,1 & 126,8 & 79 & 0,3 & 1,8 \\
\hline & Alaska 2 & 24,7 & 8,1 & $42 \%$ & 153 & 25,8 & 81,2 & 44 & 0,4 & 1,2 \\
\hline & \begin{tabular}{|l|} 
Miraflores 2 \\
\end{tabular} & 24,8 & 16,4 & $64 \%$ & 320 & 25,3 & 219,6 & 71 & 0,5 & 4,4 \\
\hline & Porvenir 2 & 31,9 & 17,2 & $52 \%$ & 207 & 32,6 & 220,6 & 61 & 0,8 & 5,3 \\
\hline \multirow{9}{*}{ Fustal } & Alaska 1 & 34,9 & 14,3 & $46 \%$ & 140 & 35,4 & 197,0 & 49 & 0,6 & 3,3 \\
\hline & Trafún 1 & 41,8 & 13,9 & $30 \%$ & 100 & 42,5 & 265,3 & 38 & 0,6 & 3,7 \\
\hline & \begin{tabular}{|l|} 
Trafun 2 \\
\end{tabular} & 39,0 & 18,4 & $54 \%$ & 160 & 39,8 & 279,3 & 60 & 0,6 & 4,3 \\
\hline & El Dorado & 36,3 & 23,9 & $57 \%$ & 233 & 36,9 & 280,4 & 62 & 0,5 & 4,0 \\
\hline & Don Cristobal & 37,1 & 36,7 & $82 \%$ & 320 & 38,1 & 554,5 & 86 & 0,5 & 8,2 \\
\hline & Puñir 2 & 31,5 & 17,2 & $70 \%$ & 213 & 32,0 & 219,6 & 73 & 0,7 & 4,8 \\
\hline & Santa Olga & 37,0 & 15,2 & $36 \%$ & 140 & 37,9 & 193,4 & 39 & 0,6 & 3,1 \\
\hline & Nueva Etruria 1 & 43,0 & 13,0 & $45 \%$ & 90 & 43,2 & 182,5 & 57 & 0,6 & 2,7 \\
\hline & Nueva Etruria 2 & 38,6 & 21,0 & $82 \%$ & 173 & 39,3 & 313,3 & 87 & 0,6 & 4,8 \\
\hline
\end{tabular}

El cuadro anterior muestra que el aporte de los árboles futuro al área basal y al volumen del rodal, oscila alrededor del $50 \%$, siendo menor en los latizales que en los fustales. En los rodales con menos de 100 árboles futuro, estos en ningún caso sobrepasan una participación en el área basal y volumen que supere el $50 \%$, encontrándose algunos muy por debajo.

\section{Análisis de Crecimiento}

El $70 \%$ de los árboles muestreados para análisis de crecimiento, mantenía un diámetro entre 25 y $45 \mathrm{~cm}$. El $87 \%$ correspondió a árboles del dosel dominante, $10 \%$ a codominantes y un 3 $\%$ al dosel intermedio.

A su vez y de acuerdo a la clasificación visual de forma del fuste, el $79 \%$ presentó forma recta, $10 \%$ torcidos, $6 \%$ curvados y el resto inclinados o bifurcados. Predominó una copa simétrica y solo un $30 \%$ de los casos correspondió a copas asimétricas. 
Finalmente, un $70 \%$ de la muestra correspondió a lo calificado como árbol futuro.

Los rodales presentaron una edad promedio de 53 años. El crecimiento promedio (IMA) para todos los rodales estuvo entre los 0,41 y $0,71 \mathrm{~cm} / a n ̃ o$, no registrándose una relación entre la edad y el crecimiento anual (Cuadro $\mathrm{N}^{\circ} 4$ ).

Cuadro $\mathrm{N}^{\circ} 4$

PARÁMETROS DE DAP, EDAD Y CRECIMIENTO DE LOS INDIVIDUOS DE ROBLE MUESTREADOS

\begin{tabular}{|l|c|c|c|c|c|c|}
\hline Rodal & $\begin{array}{c}\text { Rango } \\
\text { DAP } \\
\text { (cm) }\end{array}$ & $\begin{array}{c}\text { DAP } \\
\text { Medio } \\
\text { (cm) }\end{array}$ & $\begin{array}{c}\text { Rango } \\
\text { Edad } \\
\text { (años) }\end{array}$ & $\begin{array}{c}\text { Promedio } \\
\text { Edad } \\
\text { (años) }\end{array}$ & $\begin{array}{c}\text { Rango } \\
\text { IMA } \\
\text { (cm) }\end{array}$ & $\begin{array}{c}\text { IMA } \\
\text { Medio } \\
\text { (cm/año) }\end{array}$ \\
\hline Alaska 1 & $29,9-49,5$ & 36,2 & $53-64$ & 60 & $0,31-0,74$ & 0,54 \\
\hline Trafun 1 & $34,3-60,5$ & 48,5 & $62-75$ & 71 & $0,44-0,69$ & 0,60 \\
\hline Miraflores 1 & $19-31,3$ & 22,2 & $29-38$ & 34 & $0,45-0,78$ & 0,58 \\
\hline Curirruca 1 & $32,8-51,8$ & 41,0 & $55-74$ & 70 & $0,42-0,84$ & 0,57 \\
\hline Curirruca 2 & $35,1-70,9$ & 50,2 & $49-75$ & 71 & $0,43-0,85$ & 0,63 \\
\hline Arquilhue 1 & $31,7-66,1$ & 45,9 & $37-67$ & 63 & $0,40-0,92$ & 0,71 \\
\hline Arquilhue 2 & $29-49,3$ & 37,3 & $35-72$ & 68 & $0,36-0,93$ & 0,66 \\
\hline Porvenir 1 & $24,2-35,3$ & 30,1 & $47-55$ & 51 & $0,37-0,62$ & 0,51 \\
\hline Puñir 1 & $25,8-37,5$ & 30,7 & $37-46$ & 42 & $0,49-0,74$ & 0,60 \\
\hline Alaska 2 & $19,5-40$ & 29,8 & $23-70$ & 66 & $0,39-0,68$ & 0,56 \\
\hline Trafun 2 & $32,2-52,4$ & 38,9 & $50-69$ & 65 & $0,48-0,68$ & 0,54 \\
\hline Miraflores 2 & $23,7-33,5$ & 29,0 & $45-54$ & 50 & $0,38-0,60$ & 0,49 \\
\hline Curirruca 3 & $37,8-52,7$ & 45,0 & $66-73$ & 69 & $0,45-0,68$ & 0,58 \\
\hline El Dorado & $23-40,7$ & 30,2 & $64-74$ & 70 & $0,29-0,62$ & 0,41 \\
\hline Don Cristobal & $21,8-45,1$ & 34,9 & $57-72$ & 68 & $0,33-0,58$ & 0,47 \\
\hline Porvenir 2 & $30,7-39,9$ & 34,1 & $38-46$ & 42 & $0,44-0,86$ & 0,65 \\
\hline Puñir 2 & $19,3-35,9$ & 28,1 & $36-50$ & 46 & $0,54-0,85$ & 0,65 \\
\hline Santa Olga & $24,2-52,2$ & 38,4 & $49-67$ & 63 & $0,31-0,80$ & 0,53 \\
\hline Nueva Etruria 1 & $16,4-51,2$ & 34,6 & $49-71$ & 67 & $0,30-0,70$ & 0,48 \\
\hline Nueva Etruria 2 & $22,2-50,9$ & 37,8 & $58-69$ & 65 & $0,33-0,65$ & 0,51 \\
\hline
\end{tabular}

\section{Respuesta a Intervenciones}

De acuerdo a los resultados obtenidos, todos los rodales presentaron árboles que superaron el $25 \%$ en su tasa de cambio en el crecimiento (GC). Al menos diez rodales tenían entre 5 o 6 árboles en el mismo período que superaron el $25 \%$ de GC, lo cual indica que efectivamente a 
nivel de rodal hubo una respuesta favorable al raleo. Las tasas de cambio que superaron el $50 \%$ fueron muy poco frecuentes y no hubo casos en que más de un árbol la presentara en un mismo período. Usando este método se detectaron en la mayoría de los casos entre 2 y 3 intervenciones durante el periodo de crecimiento.

Al analizar los resultados a nivel de rodales, se determinó que las primeras intervenciones se realizaron entre los 15 y 46 años de edad, registrándose tasas de crecimiento radial promedio (GC) que variaron entre los 0,27 y 1,48. A nivel de crecimiento en DAP esto se traduce en incrementos de entre 0,22 y $0,59 \mathrm{~cm}^{(C u a d r o} \mathrm{N}^{\circ} 5$ ).

Los análisis muestran que el $45 \%$ de los rodales fueron intervenidos después de los 30 años de edad, mientras que solo el $15 \%$ de los rodales fueron intervenidos a temprana edad (antes de 20 años)

Cuadro $\mathrm{N}^{\circ} 5$

VALORES AGREGADOS DE CRECIMIENTO Y PROBABILIDAD DE INTERVENCIÓN A NIVEL DE RODALES

\begin{tabular}{|c|c|c|c|c|c|c|c|c|c|c|c|c|}
\hline \multirow[b]{2}{*}{ Rodal } & \multicolumn{3}{|c|}{ Primera Intervención } & \multicolumn{3}{|c|}{ Segunda Intervención } & \multicolumn{3}{|c|}{ Tercera Intervención } & \multicolumn{3}{|c|}{ Cuarta Intervención } \\
\hline & GC & $\begin{array}{l}\text { Edad } \\
\text { (años) }\end{array}$ & $\begin{array}{c}\text { Crec. } \\
\text { DAP (cm) }\end{array}$ & GC & $\begin{array}{l}\text { Edad } \\
\text { (años) }\end{array}$ & $\begin{array}{c}\text { Crec. } \\
\text { DAP (cm) }\end{array}$ & GC & $\begin{array}{l}\text { Edad } \\
\text { (años) }\end{array}$ & $\begin{array}{c}\text { Crec. } \\
\text { DAP }(\mathrm{cm})\end{array}$ & GC & $\begin{array}{l}\text { Edad } \\
\text { (años) }\end{array}$ & $\begin{array}{c}\text { Crec. } \\
\text { DAP }(\mathrm{cm})\end{array}$ \\
\hline Alaska 1 & 0,50 & 37 & 0,29 & 0,81 & 44 & 0,19 & 0,57 & 48 & 0,16 & & & \\
\hline Trafun 1 & 1,48 & 20 & 0,58 & 0,65 & 23 & 0,73 & 0,33 & 50 & 0,60 & 0,50 & 57,00 & 0,35 \\
\hline Miraflores 1 & 0,46 & 15 & 0,46 & 0,40 & 24 & 0,39 & & & & & & \\
\hline Curirruca 1 & 0,68 & 40 & 0,42 & & & & & & & & & \\
\hline Curirruca 2 & 0,43 & 46 & 0,40 & 0,44 & 59 & 0,39 & & & & & & \\
\hline Arquilhue 1 & 1,41 & 30 & 0,48 & & & & & & & & & \\
\hline Arquilhue 2 & 0,44 & 36 & 0,49 & & & & & & & & & \\
\hline Porvenir 1 & 0,71 & 18 & 0,41 & 0,51 & 28 & 0,43 & 0,73 & 43 & 0,31 & & & \\
\hline Puñir 1 & 0,55 & 29 & 0,46 & 0,38 & 21 & 0,52 & & & & & & \\
\hline Alaska 2 & 1,19 & 27 & 0,40 & 0,39 & 35 & 0,58 & 0,41 & 43 & 0,48 & & & \\
\hline Trafun 2 & 0,39 & 38 & 0,59 & 0,53 & 50 & 0,32 & & & & & & \\
\hline Miraflores 2 & 0,35 & 26 & 0,39 & & & & & & & & & \\
\hline Curirruca 3 & 1,04 & 46 & 0,34 & 0,36 & 36 & 0,31 & 0,46 & 61 & 0,40 & & & \\
\hline El Dorado & 0,27 & 36 & 0,33 & 0,59 & 56 & 0,24 & & & & & & \\
\hline Don Cristobal & 0,47 & 30 & 0,39 & 0,45 & 45 & 0,33 & 0,42 & 55 & 0,30 & & & \\
\hline Porvenir 2 & 0,50 & 17 & 0,59 & 0,32 & 32 & 0,51 & & & & & & \\
\hline Puñir2 & 0,39 & 28 & 0,40 & 0,49 & 32 & 0,36 & & & & & & \\
\hline Santa Olga & 0,33 & 23 & 0,38 & 0,34 & 29 & 0,48 & 0,34 & 46 & 0,23 & & & \\
\hline Nueva Etruria 1 & 1,21 & 28 & 0,22 & 1,44 & 31 & 0,28 & 0,37 & 52 & 0,33 & & & \\
\hline Nueva Etruria 2 & 0,34 & 20 & 0,55 & 0,34 & 35 & 0,42 & 0,46 & 53 & 0,35 & 0,51 & 56,00 & 0,36 \\
\hline
\end{tabular}

GC: Tasa del cambio en crecimiento radial

Edad: Edad estimada al momento de la intervención

Crec. DAP $(\mathrm{cm})$ : Crecimiento en DAP a la edad de la intervención 
Una segunda intervención se realizó a 16 rodales, los cuales tenían edades que variaron entre 21 y 59 años. Las tasas de crecimiento radial de los rodales estuvieron entre los 0,32 y 1,44, y los crecimientos en DAP producto de esta segunda intervención, varió entre $0,19 \mathrm{~cm}$ y $0,73 \mathrm{~cm}$.

Solo 9 rodales fueron intervenidos en una tercera oportunidad y esto sucedió entre los 43 y 61 años de edad. Las tasas de crecimiento radial promedio variaron entre los 0,33 y 0,73, mientras que el crecimiento en DAP estuvo en el orden de los 0,16 y $0,6 \mathrm{~cm}$.

Dos rodales fueron intervenidos por cuarta vez, ambos a una edad cercana, correspondiente a 56 y 57 años, con similares tasa promedio de 0,50 y 0,51 , y con crecimientos en DAP de 0,35 y $0,36 \mathrm{~cm}$. Ambos rodales tienen un estimado de 20 años en su primera intervención alrededor de 30 años en la segunda y 50 años en la tercera.

La oportunidad del raleo corresponde matemáticamente al punto donde el ICA es máximo (maximizar volumen individual) y antes de que la curva comience a decrecer, lo que de acuerdo a la Figura $\mathrm{N}^{\circ} 7$ sucede a los 12 años y donde el crecimiento es de $0,85 \mathrm{~cm}$. O también al momento donde el crecimiento medio anual supere al crecimiento corriente (maximizar volumen del rodal), aproximadamente a los 24 años con una IMA de $0,63 \mathrm{~cm}$.

Si bien la Figura $N^{\circ} 7$ representa una situación generalizada de los rodales, en la Figura $\mathrm{N}^{\circ} 8$, se presentan las curvas de crecimiento para un conjunto de rodales en donde se reflejan las condiciones particulares de cada uno.

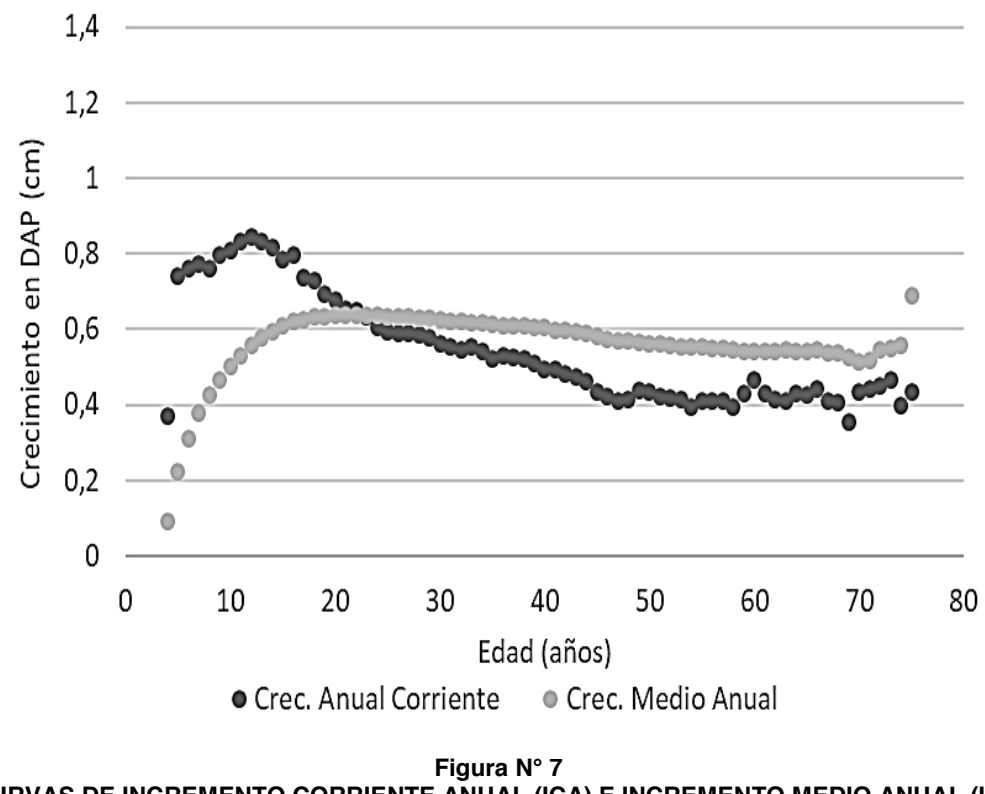

CURVAS DE INCREMENTO CORRIENTE ANUAL (ICA) E INCREMENTO MEDIO ANUAL (IMA) 

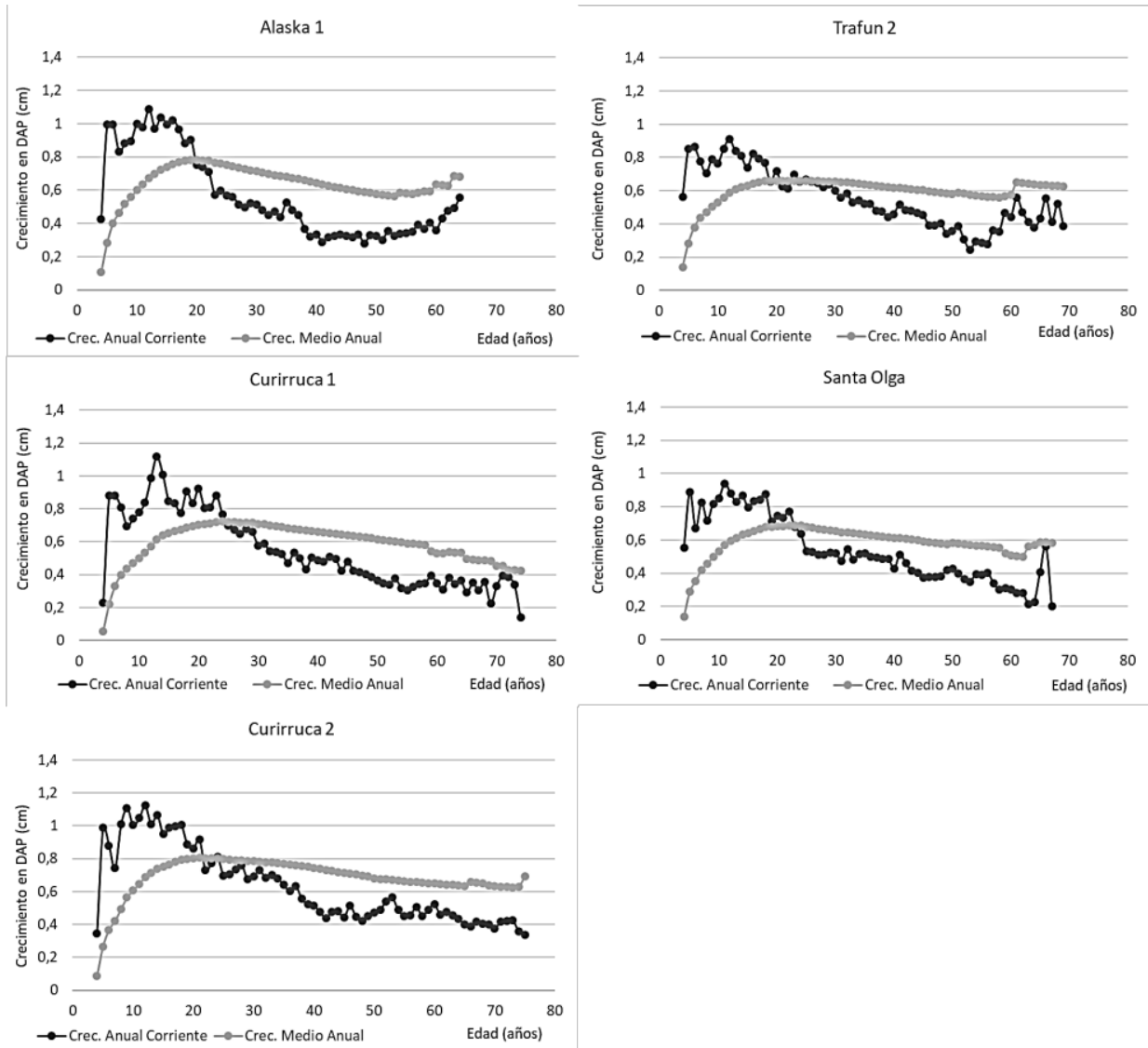

\section{Figura $\mathrm{N}^{\circ} 8$ \\ CRECIMIENTOS CORRIENTES Y MEDIOS PARA RODALES DE RESPUESTA BAJA A LAS INTERVENCIONES}

Al observar la curva de ICA de los rodales se pueden ver saltos como el del rodal Santa Olga cerca de los 65 años, o del rodal Trafun 2 alrededor de los 60 años, pero que no superan el IMA. A partir de esto, se puede deducir que la oportunidad o la intensidad de la liberación de competencia no fueron las adecuadas. En este grupo la edad en que se alcanza el máximo ICA en promedio es a los 12 años, con $1,02 \mathrm{~cm}$, y el IMA máximo a los 21 años, con $0,73 \mathrm{~cm}$. Si se observa la edad estimada de la primera intervención, todos estos rodales han comenzado a ralearse después de los 37 años, a excepción del rodal Santa Olga, que fue intervenido a una edad estimada de 22 años. La Figura $\mathrm{N}^{\circ} 9$ presenta rodales que retrasaron el cruce de curvas y la mayoría mantuvo un crecimiento cercano al IMA, y en algunos casos superior. 
Miraflores 1

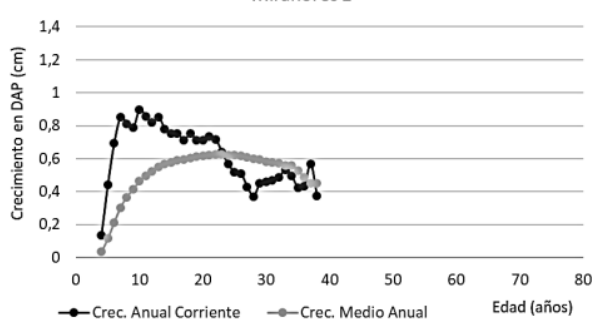

Arquilhue 1

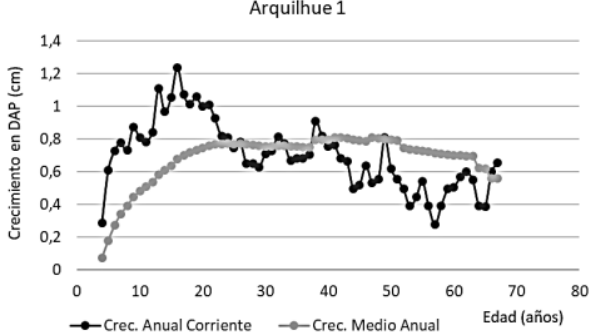

Puñir 1

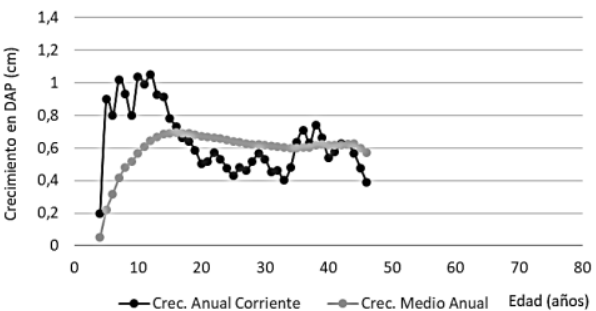

Alaska 2

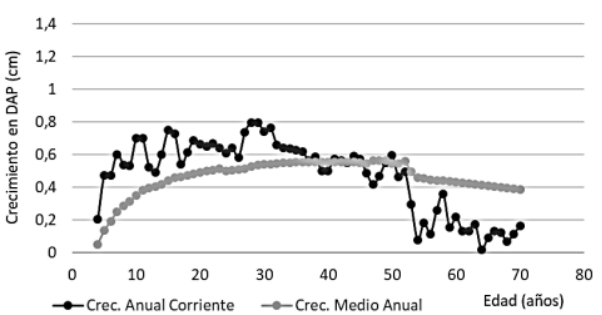

Miraflores 2

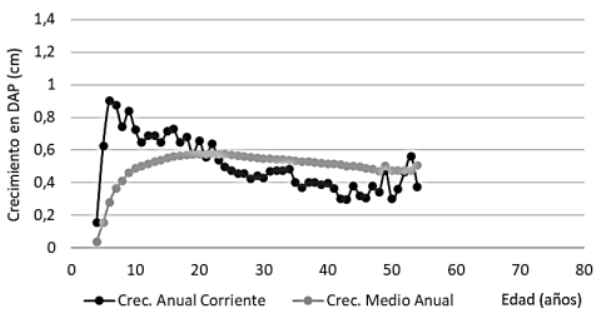

Don cristobal

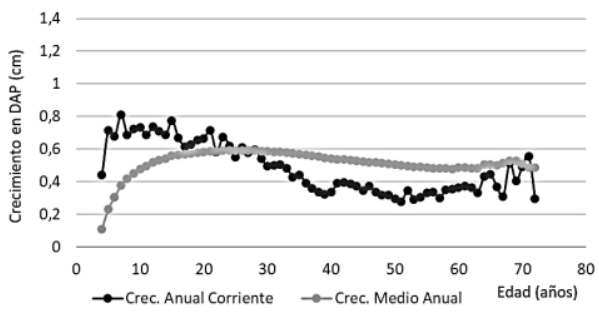

Puñir 2

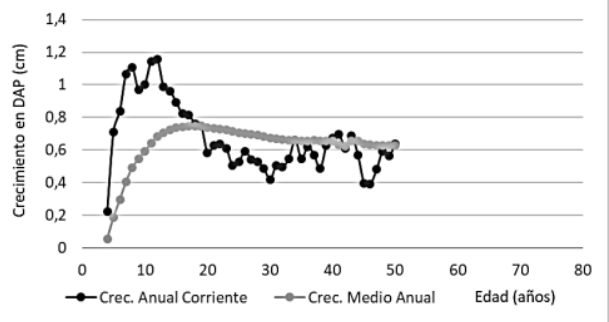

Figura $\mathrm{N}^{\circ} 9$

CRECIMIENTOS CORRIENTES Y MEDIOS PARA RODALES DE RESPUESTA INTERMEDIA A LAS INTERVENCIONES 
En estos rodales el ICA máximo se produce en promedio alrededor de los 14 años, con un valor medio de $0,94 \mathrm{~cm}$. El IMA se produce cerca de los 24 años con valores de 0,65 cm. El ICA supera al IMA nuevamente cerca de los 40 años con un valor promedio de $0,71 \mathrm{~cm}$. EI ICA del rodal Don Cristobal supera a los 71 años el IMA. En este grupo la edad de la primera intervención se estimó entre los 26 y 30 años (salvo el rodal Miraflores 1). Es decir, un poco antes que el grupo anterior.

En la Figura $\mathrm{N}^{\circ} 10$ se aprecian los casos que reflejan mejores resultados de las intervenciones al mantener su crecimiento ICA por sobre el IMA en varios períodos y ambos cercanos en su tendencia.
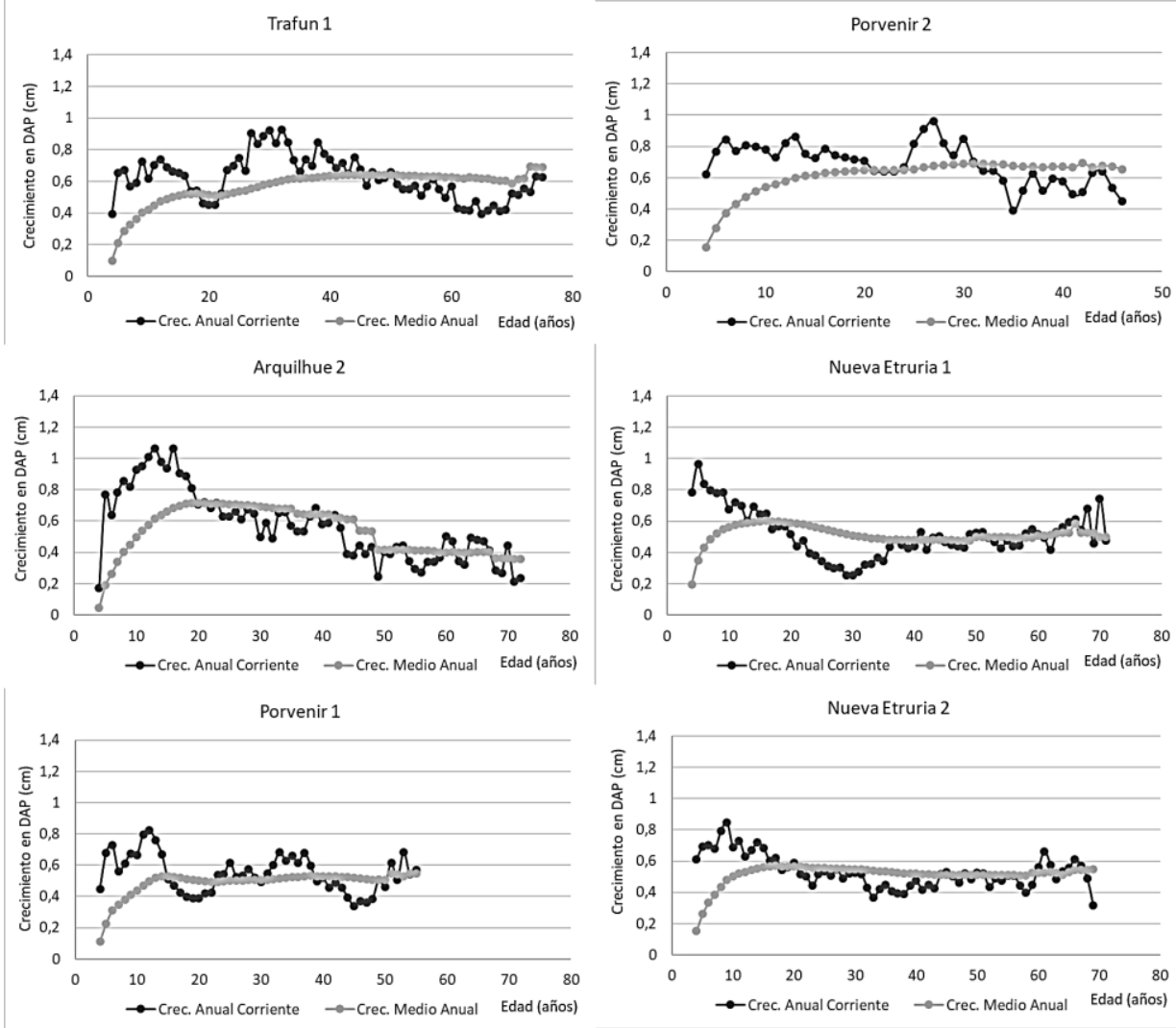

Figura $N^{\circ} 10$

CRECIMIENTOS CORRIENTES Y MEDIOS PARA RODALES CON MEJOR RESPUESTA A LAS INTERVENCIONES 
Los ICA máximos se producen en forma temprana alrededor de los 12 años y su valor alcanza $0,84 \mathrm{~cm}$ con un IMA de $0,54 \mathrm{~cm}$. A diferencia de los grupos anteriores, en estos rodales es posible observar los aumentos de los ICA, producto de la liberación de la competencia. De este grupo, los rodales Trafun 1 y Porvenir 1 presentan las primeras intervenciones entre los 17 y 20 años de edad, es decir, corresponden a los que han sido intervenidos en forma más temprana.

\section{DISCUSIÓN}

Los renovales de roble, al ser formaciones secundarias y pioneras, generalmente surgidas de forma espontánea tras fuertes disturbios o también tras el abandono de tierras agrícolas y ganaderas, constituyen naturalmente una fase corta en la sucesión natural del bosque (Donoso 1993).

Es por ello, que es frecuente encontrar situaciones de bosque de roble, donde la sucesión ya ha avanzado y se ha establecido un segundo estrato de especies semitolerantes y tolerantes. Si bien esto presenta oportunidades para la silvicultura, estas no son objeto de este estudio, que está enfocado en el análisis del crecimiento de esta primera generación de robles y en algunos casos en mezcla con raulí.

Por ser una especie pionera, roble culmina su crecimiento corriente tempranamente, entre los 10 y 15 años. Así lo indica este estudio y también lo han comprobado otros estudios relacionados (Donoso, 1993; Attis et al., 2018). De esta forma, la capacidad de respuesta al raleo en roble es muy buena si se realiza a temprana edad (Donoso, 1993).

Según estos autores, rodales jóvenes en los cuales se mantiene baja la competencia entre individuos a través de raleos, mantienen incrementos corrientes anuales en diámetro de alrededor de $0,8 \mathrm{~cm}$. Mientras que a una avanzada edad el potencial de crecimiento disminuye. Según el modelo de crecimiento propuesto por Attis et al. (2018) para los bosques de roble-raulí y coigüe en los alrededores del Lago Lacar en Argentina, roble a una edad de 50 años, alcanza solo un $80 \%$ del crecimiento máximo, y a los 100 años cerca de $50 \%$.

Gran parte de los renovales de esta especie actualmente se encuentran en un rango entre 50 y 90 años de edad, por lo que es relevante conocer en detalle su comportamiento ante el manejo.

El presente estudio ha mostrado que, en rodales bajo manejo es posible mantener un nivel de crecimiento óptimo para los árboles dominantes, también a una avanzada edad. Las tasas máximas de crecimiento corriente del presente estudio varían entre 0,82 a 1,06 cm, muy similar a los simulados por Attis et al. (2018).

Las tasas que se producen en respuesta a los últimos raleos, van desde 0,5 a 0,68 cm, o sea entre un $47 \%$ y un $83 \%$ del crecimiento máximo, para edades entre 53 y 74 años. El rodal de más edad, 74 años, alcanza todavía un $68 \%$ de su crecimiento máximo.

Cabe señalar que, si bien la primera intervención depende del estado del bosque y de las condiciones de mercado, dado que lo que se extrae del bosque puede no tener valor comercial o de un valor muy bajo para costear la operación, desde el punto de vista del producto futuro, esta primera intervención marcará las tasas de crecimiento futuras.

Desde el punto de vista biológico, los rodales se deben ralear antes de que surjan serias situaciones de competencia entre los árboles si se desea que los incrementos comerciales 
aumenten (Drake et al., 2003). Los raleos se llevan a cabo cuando se puede justificar que una aplicación aumentaría el crecimiento en volumen o los retornos económicos (Daniel et al., 1982).

De gran relevancia en relación al valor y a la efectividad de los raleos es también la cantidad de árboles de calidad con los cuales el rodal alcanza su meta de diámetro. Roble es una especie que tiene una alta capacidad de poda natural y se caracteriza por fustes rectos y cilíndricos, ambos criterios importantes para la calidad de la madera.

Sin embargo, y debido al uso tradicional del floreo (Vásquez-Grandón., 2018), es frecuente que los árboles de mejor calidad y crecimiento sean cosechados tempranamente, para generar un flujo de ingreso desde el bosque.

Los rodales que han sido sujetos a este tipo de uso pueden tener escasez de árboles de calidad para una satisfactoria producción maderera. Eso mismo puede acontecer en rodales con alta presencia de ganado, donde el efecto del ramoneo y pisoteo puede conducir a una alteración de la mezcla de especies y daños en los individuos.

Otro elemento desvalorizador y que puede llevar a una escasez de árboles futuro, es el ataque del Cerambicidae barrenador Holopterus chilensis. Cabrera (1997) muestra la alta vulnerabilidad de rodales puros de roble ante este insecto y el ataque prevalente de los árboles dominantes y codominantes, sobre todo en la parte baja del fuste, afecta en forma significativa la calidad maderera y la productividad del rodal, al ser en las primeras trozas en donde se acumula el mayor valor del arbol.

La cantidad de árboles futuro es un aspecto restrictivo que determina el potencial de madera de calidad de un rodal. Ello, considerando que es necesario mantener alrededor de 200 árboles futuro a nivel de hectárea para hacer un uso eficiente del espacio hasta el final de la rotación (un diámetro meta de $40 \mathrm{~cm}$ ), en circunstancias que este estudio determinó que solo el $30 \%$ de los rodales muestreados cumplieron con esa condición.

En rodales donde se encontró una cantidad suficiente de árboles futuro, un indicador para la efectividad con la cual los raleos han promovido el crecimiento de estos, es el indicador de Zachara (2014). Este indicador compara el diámetro medio de los árboles futuro con el diámetro medio de los 100 árboles más gruesos.

Aplicando este indicador a los rodales del presente estudio (Cuadro $\mathrm{N}^{\circ} 6$ ) se muestra que en general, los árboles futuro tienen índices superiores a 0,7, lo que de acuerdo a Zachara (2014) da cuenta de la reacción positiva de estos árboles a los raleos.

Sin embargo, se tiene que considerar que algunos rodales analizados, contaban con números bajos de árboles que cumplieran los requerimientos de calidad de un árbol futuro, a veces menos de 100 árboles/ha. Esto, eventualmente podría generar una sobreestimación en el indicador usado, fundamentalmente en aquellos valores superiores a 1 o muy cercanos a 1 . Esto reafirma que la calidad es un factor clave al momento de evaluar el efecto del raleo.

Otro indicador para el efecto positivo de los raleos analizados es que el incremento medio anual (IMA) de los árboles futuro, es mayor que el de los robles en general. 
Cuadro $N^{\circ} 6$

COEFICIENTE DE DOMINANCIA DE ÁRBOLES FUTURO

RESPECTO A LOS 100 ÁRBOLES MÁS GRUESOS

\begin{tabular}{|l|c|c|c|}
\hline Nombre & $\begin{array}{c}\text { DAP medio arboles } \\
\text { futuro } \\
\text { (cm) }\end{array}$ & $\begin{array}{c}\text { DAP medio 100 árboles más } \\
\text { gruesos } \\
\text { (cm) }\end{array}$ & Índice \\
\hline Alaska 1 & 34,9 & 44,1 & 0,79 \\
\hline Trafún 1 & 41,8 & 50,4 & 0,83 \\
\hline Miraflores 1 & 23,8 & 28,1 & 0,85 \\
\hline Curirruca 1 & 38,6 & 42,9 & 0,90 \\
\hline Curirruca 2 & 46,7 & 52,1 & 0,90 \\
\hline Arquilhue 1 & 45,9 & 40,5 & 1,13 \\
\hline Arquilhue 2 & 39,5 & 44,7 & 0,88 \\
\hline Porvenir 1 & 34,8 & 37,3 & 0,93 \\
\hline Puñir 1 & 29,2 & 33,0 & 0,88 \\
\hline Alaska 2 & 24,7 & 32,6 & 0,76 \\
\hline Trafun 2 & 39,1 & 44,9 & 0,87 \\
\hline Miraflores 2 & 24,8 & 32,8 & 0,76 \\
\hline Curirruca 3 & 19,3 & 29,7 & 0,65 \\
\hline El Dorado & 36,3 & 43,3 & 0,84 \\
\hline Don Cristobal & 37,1 & 48,4 & 0,77 \\
\hline Porvenir 2 & 31,9 & 38,5 & 0,83 \\
\hline Puñir 2 & 31,5 & 37,0 & 0,85 \\
\hline Santa Olga & 37,0 & 43,9 & 0,84 \\
\hline Nueva Etruria 1 & 43,0 & 45,6 & 0,94 \\
\hline Nueva Etruria 2 & 38,6 & 43,5 & \begin{tabular}{c} 
(n, \\
\hline
\end{tabular} \\
\hline
\end{tabular}

\section{CONCLUSIONES}

En general, la cantidad de árboles futuro que concentran el potencial maderero de los rodales es un aspecto a tener en consideración, puesto que solo el $30 \%$ de los rodales muestreados mantiene una cantidad de individuos futuro superior a 200 árboles/ha. Si se piensa en un diámetro de cosecha de $40-45 \mathrm{~cm}$ luego de una rotación para roble, entonces existiría un déficit de árboles futuro en la mayoría de estos rodales.

A partir de los resultados obtenidos resulta claro que los rodales seleccionados han respondido a las intervenciones realizadas. El método utilizado ha permitido aproximarse a los períodos de intervenciones pasadas y determinar la respuesta a las intervenciones. Tras el análisis 
de los crecimientos corrientes y medios se destaca que los crecimientos corrientes máximos se presentan alrededor de los 12 años, en los distintos rodales medidos y los IMA máximos bordean los 20 años.

El análisis gráfico de las curvas de crecimiento permite diferenciar tres tipos de comportamientos. Aquellos cuyo crecimiento corriente se mantiene gran parte del desarrollo del rodal bajo el crecimiento medio, que además coinciden con aquellos que comenzaron más tardíamente las intervenciones, y que probablemente por oportunidad o posiblemente intensidad no logran revertir sus tasas de crecimiento. Un segundo grupo que muestra una tendencia a mantener ambos incrementos muy cercanos y demorar la disminución en su tasa. Este grupo coincide con aquellos rodales que se intervinieron más tempranamente que los anteriores. Un tercer grupo que a través del tiempo presenta mejores tasas de crecimiento y que son los rodales que primero se han intervenido.

Los resultados obtenidos demuestran la necesidad de iniciar las primeras intervenciones o raleos a partir de los 12 años y antes de los 20 años. Cuando los rodales son raleados tempranamente mantienen su potencial de crecimiento aun en las edades superiores. Se estima que, en los últimos raleos, un rodal puede alcanzar entre el $47 \%$ y un $83 \%$ de su crecimiento máximo para edades entre 53 y 74 años.

Una intervención temprana, a pesar de no ser atractivao desde el punto de la extracción de volumen maderero e ingreso económico, y quizás no logre cubrir los costos de la intervención, sin duda determinará una mejor tasa de crecimiento futura del rodal.

\section{REFERENCIAS}

Abrams, M. D.; Copenheaver, C. A.; Black, B. A. and Van de Gevel, S., 2001.Dendroecology and climatic impacts for a relict, old-growth, bog forest in the Ridge and Valley Province of central Pennsylvania, USA.Can. J. Bot. 79: 58-69.

Assmann, E., 1970. The principles of forest yield study, Pergamon Press, Oxford.

Attis, Beltran; Chauchard, H.; Dezzotti, L.; y Martinez Pastur, G., 2018. Modelo de crecimiento diamétrico de Nothofagus alpina y su relación con el de Nothofagus obliqua y Nothofagus dombeyi en los bosques naturales de la Patagonia argentina. Bosque 39(1): 107-117.

Altman, J.; Fibich, P.; Dolezal, J. y Aakala, T., 2014. TRADER: A package for tree ring analysis of disturbance events in R. Dendrochronologia 32, 107-112.

Black, B. A. and Abrams, M. D., 2003. Use of boundary-line growth patterns as a basis for dendroecological release criteria. Ecol. Appl. 13:1733-1749.

Cabrera, P., 1997. Impacto de Holopterus chilensis (Coleoptera: Cerambycidae) en renovales de Nothofagus obliqua en la provincia de Valdivia, Chile. Revista Bosque 18(1) 9-19.

CONAF, 2017. Sistema de Información Territorial (SIT). Corporación Nacional Forestal (CONAF). Ministerio de Agricultura. Consultado el 20 de abril 2020. Disponible en http://sit.conaf.cl/

CONAF, 2018. Consideraciones para la Formulación del Plan de Manejo Forestal de Bosque Nativo. Ley $\mathrm{N}^{\circ}$ 20.283. Disponible en https://www.conaf.cl/wp-content/uploads/2012/12/Consideraciones-formulacionPMFBN_Version-Enero-2018.pdf. Visitado en julio 22, 2020.

Daniel, T.; Helms, J. A. y Backer, F. S., 1982. Principios de silvicultura. McGraw-Hill. México. 492p. 
Donoso, C., 1993. Bosques templados de Chile y Argentina. Variación, estructura y dinámica. Santiago, Chile. Editorial Universitaria. $483 p$

Donoso, P.; Donoso, C. y Sandoval, V., 1993. Proposición de zonas de crecimiento de renovales de Roble (Nothofagus obliqua) y Raulí (Nothofagus alpina) en su rango de distribución natural. Revista Bosque 14(2):37-49.

Drake, F.; Acuna, E.; Salas, S., 2003. Evaluación retrospectiva para determinar la oportunidad de raleo en un rodal de Pino Oregón de 24 años. Revista Bosque 24(2): 85-91.

FAO, 2016. Casos ejemplares de manejo forestal sostenible en Chile, Costa Rica, Guatemala y Uruguay. Santiago, Chile $246 \mathrm{p}$

Fraver, S. and White, A. S., 2005. Identifying growth releases in dendrochronological studies of forest disturbance. Canadian Journal of Forest Research 35:1648-1656.

Gezan, S.; Ortega, A y Andenmatten, E., 2007. Diagramas de manejo de densidad para renovales de roble, raulí y coigüe en Chile. Revista Bosque 28(2): 97-105.

Grosse, H. y Villalobos, E., 2009. Silvicultura del Bosque nativo chileno - Función histórica y opciones futuras sobre la base de Manejo sustentable. Santiago $135 \mathrm{p}$.

Grosse, H.; Larrain, O. y Mujica, R., 2007. Valorización de los bosques de segundo crecimiento para los tipos forestales roble-raulí-coigüe y coigüe-raulí-tepa. Ciencia e Investigación Forestal 13(2): 371-384.

Grosse, H. y Quiroz, I., 1999. Silvicultura de los bosques de segundo crecimiento de roble, raulí y coigüe en la región centro-sur de Chile. p 95-125. Silvicultura de los bosques nativos de Chile. Editorial Universitaria. 421p.

INFOR, 2018. Bosque Nativo. Boletín No 15. junio de 2018. 20 p.

Juacida, R.; Siebert, H. y Torres, M., 2000. Edad y durabilidad natural de renovales de Nothofagus obliqua. Revista Bosque 21(1): 73-7.

Lavery, P. B., 1986. Plantation forestry with Pinus radiata: review papers. Paper $\mathrm{N}^{\circ} 12$. School of Forestry, Univ. of Canterbury. Christchurch, New Zealand.

Lorimer, C.G. y Frelich, L. E., 1989. A Methodology for Estimating Canopy Disturbance Frequency and Intensity in Dense Temperate Forests. Can. J. For. Res. 19: 651-663.

Martin, M.; Bahamóndez, C.; Quiróz, I.; Peña, O. y Barrientos, M., 2009. Manual de operaciones en terreno. Inventario de los ecosistemas forestales. Ed. Bahamóndez, C. Instituto Forestal, Chile. Manual N²0. 139 p.

Müller-Using, S.; Martin, M.; Müller-Using, B.; Grosse, H. y Mujica, R., 2012. Estado actual y propuestas silvícolas para los renovales de Nothofagus en la zona centro sur de Chile. Reporte Técnico 190. INFOR.

Nowacki, G. J. and Abrams, M. D., 1997. Radial-growth averaging criteria for reconstructing disturbance histories from presettlement-origin oaks. Ecol. Monogr. 67: 225-249.

Peralta, M., 1976. Uso, clasificación y conservación de suelos. Santiago, Ministerio de Agricultura. Servicio Agrícola y Ganadero. 337 p.

Trotsiuk, V.; Pedersond, N.; Druckenbrode, D.; Orwig, D.; Bishop, D.; Barker-Plotkind, A.; Fraverh, S. and Martin-Benito, D., 2018. Testing the efficacy of tree-ring methods for detecting past disturbances. Forest Ecology and Management 425: 59-67.

Vásquez-Grandón, A.; Donoso, P. y Gerding, V., 2018. Degradación de los bosques: Concepto, proceso y estado - Un ejemplo de aplicación en bosques adultos nativos de Chile. En: Silvicultura en bosques nativos: Experiencias en Silvicultura y restauración en Chile, Argentina y el oeste de Estados Unidos. Editores: P. Donoso, A. Promis, D. Soto. 176-196. 
Veblen, T. T.; Donoso, C.; Schlegel, F. M. and Escobar, B., 1981. Forest dynamics in south-central Chile. Journal of Biogeography 8(3): 211-247.

Zachara, T., 2014. The influence of different thinning methods on dominance coefficients of future crop trees in even-aged Scots Pine stands. Forest Research Papers | Volume 75: Issue 1 DOI: https://doi.org/10.2478/frp2014-0004 
